\title{
Estudio comparativo de perfil lipídico y presión arterial en cani- nos delgados y con sobrepeso
}

\author{
Comparative study of lipidic profile and blood pressure in lean and \\ overweight dogs
}

\author{
Silvana Garaycochea C..,4, Roberto Dávila F. ${ }^{1}$, Boris Lira M. ${ }^{2}$, Francisco Suárez A. ${ }^{3}$
}

\section{Resumen}

\begin{abstract}
El objetivo del presente estudio fue comparar los niveles de colesterol, triglicéridos y presión arterial en caninos. Se trabajó con 60 caninos, de los cuales 30 eran delgados y 30 tenían sobrepeso. Los animales eran pacientes de la Clínica de Animales Menores de la Facultad de Medicina Veterinaria de la Universidad Nacional Mayor de San Marcos y eran libres de enfermedades cardiacas. Las muestras de sangre colectadas fueron procesadas mediante fotocolorimetría en un analizador químico y la medida de la presión arterial se hizo con un tensiómetro digital. Los resultados demostraron una hipertrigliceridemia en el $16.7 \%$ del grupo sobrepeso, mientras que el $46.7 \%$ del grupo delgado y el $90 \%$ del grupo sobrepeso mostraron hipercolesterolemia. El 20 y $50 \%$ de los canes del grupo delgado y sobrepeso, respectivamente, registraron valores de hipertensión arterial. Los niveles de triglicéridos y colesterol fueron significativamente superiores en el grupo sobrepeso $(p<0.05)$, mientras que no hubo diferencias en los valores de la presión arterial sistólica. Se encontró una asociación entre los caninos delgados y con sobrepeso con las variables triglicéridos y colesterol.
\end{abstract}

Palabras clave: caninos; delgado; sobrepeso; colesterol; triglicéridos; presión arterial

\section{AbSTRACT}

The aim of the present study was to compare levels of cholesterol, triglycerides, and blood pressure in canines. A total of 60 dogs were used (30 were lean and 30 were overweight). The animals were patients from the Clinic of Small Animals of the Faculty of Veterinary Medicine of the National University of San Marcos and were free of heart diseases. Blood samples were collected and processed by photocolorimetry in a chemical

\footnotetext{
${ }^{1}$ Clínica de Animales Menores, ${ }^{2}$ Laboratorio de Fisiología Animal, ${ }^{3}$ Laboratorio de Medicina Veterinaria Preventiva, Facultad de Medicina Veterinaria, Universidad Nacional Mayor de San Marcos, Lima, Perú

${ }^{4}$ E-mail: silvana.garayco648@outlook.com
}

Recibido: 6 de marzo de 2017

Aceptado para publicación: 10 de julio de 2018 
analyzer and the blood pressure measurement was done with a digital blood pressure monitor. The results showed hypertriglyceridemia in $16.7 \%$ of the overweight group, while $46.7 \%$ of the lean group and $90 \%$ of the overweight group showed hypercholesterolemia. In addition, 20 and $50 \%$ of the lean and overweight group respectively had high blood pressure values. Triglyceride and cholesterol levels were significantly higher in the overweight group $(\mathrm{p}<0.05)$, whereas there were no differences in systolic blood pressure values. There was an association between lean and overweight dogs with triglycerides and cholesterol.

Key words: canine; lean; overweight; cholesterol; triglycerides; blood pressure

\section{INTRODUCCIÓN}

La obesidad canina es el desorden nutricional más importante que se presenta en los animales de compañía. Los perros obesos tienden a padecer problemas de salud, tales como dislipidemias, hipertensión arterial, diabetes, hiperadrenocorticismo e hipotiroidismo (Zhang y Reisen, 2001; Cavalieri, 2012). Condiciones de hipertensión e hiperlipidemia han sido reportadas en animales con sobrepeso (Oyarzo, 2010; Brunetto et al., 2011); sin embargo, en el país no se dispone de rangos de valores normales de presión arterial, triglicéridos ni colesterol en caninos que puedan avalar estas condiciones.

La hiperlipidemia se relaciona con el desarrollo de enfermedades como pancreatitis, aterosclerosis y lesiones oculares (Jeusette et al., 2005). La hipertensión puede llegar a desencadenar fallas orgánicas relacionadas, principalmente, a una alteración funcional a nivel renal y cardiaco (Brown et al., 2007). La repercusión de estas condiciones en la obesidad canina aún sigue siendo estudiada (Brunetto et al, 2011; Pérez et al., 2015).

El objetivo del presente estudio fue determinar y comparar el perfil lipídico y presión arterial entre caninos delgados y con sobrepeso en la zona de Lima. Los resultados permitirán establecer rangos de presión arterial, triglicéridos y colesterol en caninos delgados y con sobrepeso.

\section{Materiales y Métodos}

El estudio se llevó a cabo en el área de cardiología de la Clínica de Animales Menores (CAMe) de la Facultad de Medicina Veterinaria (FMV) de la Universidad Nacional Mayor de San Marcos (UNMSM), Lima, Perú, entre marzo de 2015 y marzo de 2016.

El cálculo del tamaño muestral se hizo mediante la fórmula de comparación de medias. Se seleccionaron 60 caninos pacientes de la CAMe, los cuales fueron clasificados por su condición corporal, según Tams (2005). De estos, 30 fueron considerados como delgados (condición corporal [CC] 3/5) y 30 con condición de sobrepeso (CC 4/5 y 5/5). La selección se realizó mediante una campaña cardiológica gratuita realizada en la clínica. Los perros que fueron inscritos por sus dueños debían estar clínicamente sanos; es decir, que no presentasen enfermedad cardiaca $\mathrm{u}$ otra que pudiera alterar las variables del estudio. No se discriminó por edad, raza o sexo. Todos los pacientes fueron examinados para determinar su CC y estado de salud antes de entrar al estudio.

Cada grupo estuvo conformado por 16 machos y 14 hembras con un rango de edades entre 6 meses y 16 años. Las razas que predominaron en el grupo delgado fueron los mestizos $(n=9)$, seguido de las razas Schnauzer y Labrador $(\mathrm{n}=4 \mathrm{c} / \mathrm{u})$. Las razas que predominaron en el grupo sobrepeso fueron Cocker $(\mathrm{n}=12)$, seguido de los mestizos $(n=7)$ y Pequinés $(n=4)$. 
La medición de presión arterial de cada paciente se realizó en el consultorio de cardiología de la CAMe. Para esto, al paciente se le daba un tiempo de 10 minutos para que se familiarice con el entorno y muestre tranquilidad. La medición se realizó en la posición decúbito lateral derecho, tomando la presión en el metatarso con el tensiómetro digital petMap (EEUU). Se realizaron cinco mediciones por paciente, escogiéndose la de menor valor para los efectos del estudio. Se consideró $160 \mathrm{mmHg}$ como límite superior de normalidad (Brown et al., 2007).

Para la medición de triglicéridos y colesterol total se tomaron muestras de sangre de la vena cefálica o safena, considerando un ayuno de $12 \mathrm{~h}$. Las muestras se procesaron en el Laboratorio de Fisiología Animal de la FMV-UNMSM, mediante el método de fotocolorimetría con el analizador de bioquímica BS 3000M (SINNOWA, China). Se consideró $112 \mathrm{mg} / \mathrm{dl}$ como límite superior de normalidad de triglicéridos y $270 \mathrm{mg} / \mathrm{dl}$ como límite superior de normalidad de colesterol (Kaneko et al., 2009).

Se utilizó la prueba de Shapiro-Wilk para determinar la normalidad de las variables. Se determinó que la variable presión arterial sistólica sigue la distribución normal, mientras que las variables triglicéridos y colesterol no presentaban normalidad. Con base a esto, se utilizó la prueba de t-Student para la variable presión arterial sistólica y la prueba de Mann-Whitney para las variables triglicéridos y colesterol. Para verificar la existencia de asociación entre las variables de estudio y el tipo de CC se utilizó la prueba de Chi cuadrado. El análisis de datos se realizó mediante el Software SPSS Statistics v. 22.0.

\section{Resultados y Discusión}

La media aritmética de triglicéridos para el grupo delgado fue de $48.8 \pm 15.3 \mathrm{mg} / \mathrm{dl} \mathrm{y}$ $95.4 \pm 109.5 \mathrm{mg} / \mathrm{dl}$ para el grupo sobrepeso $(p<0.05)$. La media del colesterol fue de
$288.9 \pm 86.6 \mathrm{mg} / \mathrm{dl}$ para el grupo delgado y $540.1 \pm 267.4 \mathrm{mg} / \mathrm{dl}$ para el grupo sobrepeso $(\mathrm{p}<0.05)$. La media de la presión arterial sistólica fue de $146.1 \pm 23.2 \mathrm{mmHg}$ para el grupo delgado y $154.83 \pm 24.18 \mathrm{mmHg}$ para el grupo sobrepeso, no encontrando diferencia estadística significativa entre grupos (Cuadro 1).

Para la variable triglicéridos, todos los perros del grupo delgado presentaron valores menores a $112 \mathrm{mg} / \mathrm{dl}$, mientras que en el grupo sobrepeso, el $16.7 \%$ presentó valores mayores de $112 \mathrm{mg} / \mathrm{dl}$ (Cuadro 2). Para la variable colesterol, el $46.7 \%$ del grupo delgado presentó valores por encima de $270 \mathrm{mg} / \mathrm{dl}$, mientras que el $90 \%$ del grupo sobrepeso presentó estos valores (Cuadro 3).

En general, los valores de triglicéridos y colesterol en el grupo con sobrepeso fueron mayores que aquellos en el grupo delgado; resultados que concuerdan con los hallados por Peña et al. (2007). Según los rangos de normalidad de perfil lipídico presentado por Kaneko et al. (2009), se podría atribuir una dislipidemia en los pacientes con registros por encima de $270 \mathrm{mg} / \mathrm{dl}$ de colesterol y de 112 $\mathrm{mg} / \mathrm{dl}$ de triglicéridos. Con base a esto, se puede indicar que 14 caninos del grupo delgado y 27 del grupo sobrepeso presentaron niveles de colesterol por encima de lo establecido, y que cinco caninos del grupo sobrepeso presentaron niveles de triglicéridos superiores al valor de referencia. Según estos resultados, se podría asumir hipercolesterolemia e hipertrigliceridemia a favor del grupo sobrepeso.

Los niveles de presión arterial sistólica fueron mayores para el grupo sobrepeso en comparación con el grupo delgado, obteniéndose una media de $154.83 \pm 24.18 \mathrm{mmHg}$ para el grupo sobrepeso y $146.1 \pm 23.23 \mathrm{mmHg}$ para el grupo delgado, aunque sin diferencia significativa entre grupos; similar a los resultados de Pérez et al. (2015). No obstante, los resultados del presente estudio contrastan con el reporte de Mehlman et al. (2013), quienes encuentran un significativo incremento en la 
Cuadro 1. Distribución (media \pm desviación estándar) de las variables triglicéridos, colesterol y presión arterial sistólica en los grupos de canes considerados como delgados y con sobrepeso con base a la condición corporal (1-5)

\begin{tabular}{lccc}
\hline & $\begin{array}{c}\text { Triglicéridos } \\
(\mathrm{mg} / \mathrm{dl})\end{array}$ & $\begin{array}{c}\text { Colesterol } \\
(\mathrm{mg} / \mathrm{dl})\end{array}$ & $\begin{array}{c}\text { Presión sistólica } \\
(\mathrm{mmHg})\end{array}$ \\
\hline Delgados $(3 / 5)$ & $48.8 \pm 15.3^{\mathrm{a}}$ & $288.9 \pm 86.6^{\mathrm{a}}$ & $146.1 \pm 23.2^{\mathrm{a}}$ \\
Sobrepeso $(4 / 5,5 / 5)$ & $95.4 \pm 109.5^{\mathrm{b}}$ & $540.1 \pm 267.4^{\mathrm{b}}$ & $154.8 \pm 24.2^{\mathrm{a}}$ \\
\hline
\end{tabular}

$a, b$ Superíndices diferentes dentro de columnas indican diferencia estadística $(p<0.05)$

Cuadro 2. Frecuencia absoluta (n) y relativa (\%) de la variable triglicéridos en el grupo delgado y el grupo sobrepeso de perros, según valores referenciales ${ }^{1}$

\begin{tabular}{ccccc}
\hline Triglicéridos & \multicolumn{2}{c}{ Delgado } & \multicolumn{2}{c}{ Sobrepeso } \\
\cline { 2 - 5 }$(\mathrm{mg} / \mathrm{dl})$ & $\mathrm{n}$ & $\%$ & $\mathrm{n}$ & $\%$ \\
\hline $20-112$ & 30 & 100.0 & 25 & 83.3 \\
$>112$ & 0 & 0 & 5 & 16.7 \\
\hline
\end{tabular}

${ }^{1}$ Kaneko et al. (2009)

Cuadro 3. Frecuencia absoluta (n) y relativa (\%) de la variable colesterol en el grupo delgado y el grupo sobrepeso de perros, según valores referenciales ${ }^{1}$

\begin{tabular}{ccccc}
\hline \multirow{2}{*}{$\begin{array}{c}\text { Colesterol } \\
(\mathrm{mg} / \mathrm{dl})\end{array}$} & $\mathrm{n}$ & $\%$ & $\mathrm{n}$ & $\%$ \\
\cline { 2 - 5 } & 16 & 53.3 & 3 & 10.0 \\
$135-270$ & 14 & 46.7 & 27 & 90.0 \\
\hline 270 & & &
\end{tabular}

${ }^{1}$ Kaneko et al. (2009)

Cuadro 4. Frecuencia absoluta (n) y relativa (\%) de la variable presión arterial en el grupo delgado y el grupo sobrepeso de perros, según valores referenciales ${ }^{1}$

\begin{tabular}{ccccc}
\hline \multirow{2}{*}{$\begin{array}{c}\text { Presión arterial sistólica } \\
(\mathrm{mmHg})\end{array}$} & $\mathrm{n}$ & $\%$ & $\mathrm{n}$ & $\%$ \\
\cline { 2 - 5 } Delgado & \multicolumn{2}{c}{ Sobrepeso } \\
\hline$<160$ & 24 & 80 & 15 & 50 \\
$=160$ & 6 & 20 & 15 & 50 \\
\hline
\end{tabular}

${ }^{1}$ Brown et al. (2007) 
Cuadro 5. Frecuencia absoluta (n) y relativa (\%) de la variable presión arterial en el grupo delgado y el grupo sobrepeso de perros, clasificadas según el riesgo de daño a corazón, riñón, ojos y cerebro ${ }^{1}$

\begin{tabular}{|c|c|c|c|c|c|}
\hline \multirow{2}{*}{$\begin{array}{l}\text { Presión arterial } \\
\text { sistólica } \\
(\mathrm{mmHg})\end{array}$} & \multicolumn{2}{|c|}{ Delgado } & \multicolumn{2}{|c|}{ Sobrepeso } & \multirow{2}{*}{$\begin{array}{l}\text { Riesgo de daño } \\
\text { orgánico }\end{array}$} \\
\hline & $\mathrm{n}$ & $\%$ & $\mathrm{n}$ & $\%$ & \\
\hline$<150$ & 20 & 66.67 & 13 & 43.33 & Mínimo \\
\hline $150-159$ & 5 & 16.67 & 2 & 6.67 & Leve \\
\hline $160-179$ & 3 & 10 & 9 & 30 & Moderado \\
\hline$\geq 180$ & 2 & 6.67 & 6 & 20 & Severo \\
\hline
\end{tabular}

${ }^{1}$ Brown et al. (2007)

presión arterial sistólica en perros con sobrepeso en comparación con perros delgados.

Según la clasificación de presión arterial presentada por Brown et al. (2007) se atribuye un cuadro hipertensivo a pacientes que registren valores de presión arterial sistólica a partir de $160 \mathrm{mmHg}$ (considerado como daño orgánico moderado y signo de hipertensión sistémica). Es así que se puede mencionar que seis canes del grupo delgado $(20 \%)$ y 15 del grupo sobrepeso $(50 \%)$ presentaron valores por encima del establecido (Cuadro 4). Estos valores podrían ser indicativos de hipertensión pero sería necesario realizar exámenes complementarios para confirmar la condición.

El $6.67 \%$ del grupo delgado presentó valores iguales o mayores a $180 \mathrm{mmHg}$, mientras que el $20 \%$ del grupo sobrepeso presentó estos valores. Según la clasificación de presión arterial propuesta por Brown et al. (2007), se distribuyeron los valores de presión arterial sistólica de ambos grupos de estudio en las categorías de mínimo, leve, moderado o severo (Cuadro 5). Los resultados indican que el riesgo de daño orgánico era de grado severo en estos animales. Los animales con sobrepeso que registraron estos valores superaron en tres veces los valores de los animales delgados, pudiendo asociarse la condición sobrepeso a niveles altos de presión arterial sistólica.

Los resultados del Chi cuadrado determinaron una asociación entre las variables de estudio y el tipo de condición corporal; sin embargo, no hubo diferencia estadística significativa en la presión arterial sistólica. Esto se debe a variaciones de la muestra como valores extremos de las variables, factores externos como ambiente, raza, sexo y el tamaño muestral.

El grupo sobrepeso estuvo conformado en su mayoría por la raza Cocker $(\mathrm{n}=12)$. Según Lund et al. (2006) y Zoran (2010), esta raza presenta una predisposición al sobrepeso. Por otro lado, se pudo determinar que 18 canes de este grupo eran alimentados con dieta mixta (balanceada más casera), lo cual implica un porcentaje de carbohidratos y lípidos adicional a sus requerimientos en la ración diaria, condicionando la elevación de los niveles de colesterol y triglicéridos. La hipercolesterolemia se registró en un mayor porcentaje en ambos grupos a diferencia de la hipertrigliceridemia, lo cual también se puede explicar por el tipo de dieta que recibía cada grupo, ya que 12 canes del grupo delgado también eran alimentados con una dieta mixta. 


\section{Conclusión}

Los caninos con sobrepeso presentaron valores altos de presión arterial y niveles altos de triglicéridos y colesterol en comparación con caninos delgados $(\mathrm{p}<0.05)$.

\section{Literatura Citada}

1. Brown S, Atkins C, Bagley R, Carr A, Cowgill L, Davidson M, Egner B, et al. 2007. Guidelines for the identification, evaluation, and management of systemic hypertension in dogs and cats. J Vet Intern Med 21: 542-558.

2. Brunetto M, Nogueira S, Sá F, Peixoto M, Souza R, Ferraudo A, Cavalieri A. 2011. Correspondencia entre obesídade e hiperlipídemia em caes. Cienc Rural 41: 266-271. doi: 10.1590/S010384782011005000004

3. Cavalieri CA. 2012. Obesidade e suas conseqüências metabólicas e inflamatórias em cães e gatos. Faculdade de Ciências Agrárias e Veterinárias da UNESP, Jaboticabal. [Internet]. Disponible en: http://www.fcav.unesp.br/ Home/departamentos/clinicacv/ AULUSCAVALIERICARCIOFI/ obesidade-texto.pdf

4. Jeusette IC, Lhoest ET, Istasse LP, Diez MO. 2005. Influence of obesity on plasma lipid and lipoprotein concentrations in dogs. Am J Vet Res 66: 81-86.

5. Kaneko JJ, Harvey JW, Bruss ML. 2009. Clinical biochemistry of domestic animals. $6^{\text {th }}$ ed. San Diego, USA: Academic Press. 904 p.
6. Lund EM, Armstrong PJ, Kirk CA, Klausner JS. 2006. Prevalence and risk factors for obesity in adult dogs from private US veterinary practices. Intern J Appl Res Vet Med 4: 177-186.

7. Mehlman E, Bright JM, Jeckel K, Porsche C, Veeramachaneni DNR, Frye M. 2013. Echocardiographic evidence of left ventricular hypertrophy in obese dogs. J Vet Intern Med 27: 62-68.

8. Oyarzo AM. 2010. Valores de presión arterial y concentraciones sanguíneas de úrea, creatinina y glucosa en perros con sobrepeso. Tesis de Médico Veterinario. Valdivia, Chile: Univ Austral de Chile. $34 \mathrm{p}$.

9. Peña C, Suárez L, Bautista I, Montoya JA, Juste MC. 2007. Relationship between analytic values and canine obesity. J Anim Physiol Anim Nutr 92: 324-325. doi: 10.1111/j.14390396.2007.00786.x

10. Pérez SA, Del Angel CJ, Quijano HI, Barbosa MM. 2015. Obesityhypertension and its relation to other diseases in dogs. Vet Res Commun 39: 45-51. doi: 10.1007/s11259-015-9630-9

11. Tams TR. 2005. Manual de gastroenterología en animales pequeños. $2^{\mathrm{a}}$ ed. Buenos Aires: Inter-Médica. 438 p.

12. Zhang R, Reisin E. 2001. Obesidadhipertensión: Efectos sobre los sistemas cardiovascular y renal. Am J Health (Ed. Esp.) 3: 150-156.

13. Zoran DL. 2010. Obesity in dogs and cats: a metabolic and endocrine disorder. Vet Clin North Am Small Anim Pract 40: 221-239. doi: 10.1016/j.cvsm.2009.10.009 